\title{
Fecal microbiota transplantation for recurrent Clostridium difficile infection
}

\author{
Susy S. Hota MD MSc, Susan M. Poutanen MD MPH
}

- Cite as: CMAJ 2018 June 18;190:E746. doi: 10.1503/cmaj.171454

CMAJ Podcasts: author interview at https://soundcloud.com/cmajpodcasts/171454-five

\section{1}

\section{Fecal microbiota transplantation is an effective treatment for recurrent Clostridium difficile infection}

Multiple randomized controlled trials show that fecal microbiota transplantation is $44 \%-96 \%$ effective in resolving recurrent $C$. difficile infection in patients with two or more recurrences. ${ }^{1}$ This is comparable or superior to conventional treatment with vancomycin taper and pulse, which is about $60 \%$ effective. ${ }^{2}$ Caution should be used in administering fecal microbiota transplantation to immunocompromised and severely ill patients, because safety has not been well-established in these groups.

\section{Most fecal microbiota transplantations are performed by} gastroenterologists and infectious disease specialists In Canada, fecal microbiota transplantations can be performed by any health care practitioner who is licensed to treat patients with a prescription drug. ${ }^{3}$ However, owing to the expertise required for donor screening, diagnosing recurrent $C$. difficile infection and providing fecal microbiota transplantation, most of these treatments are provided by specialists who have specific interest in fecal microbiota transplantation.

\section{3}

\section{Donors should be rigorously screened}

Health Canada provides rigorous donor-screening guidance focused on minimizing risks of bloodborne, enteric and sexually transmitted infection. ${ }^{3}$ Some fecal microbiota transplantation programs additionally exclude donors with personal or family histories of diseases associated with disrupted intestinal microbiota, such as obesity and depression. At a large US stool bank, less than $3 \%$ of prospective donors pass screening. ${ }^{4}$

\section{There are four ways to administer fecal microbiota transplantation}

Fecal microbiota can be delivered by enema, colonoscopy or through a nasoduodenal tube. Capsules are under development but are not yet commercially available. Currently, no data suggest superiority of one route of administration over another. ${ }^{1}$

\section{5}

\section{The long-term health effects are unknown}

Although short-term safety in resolving recurrent $C$. difficile infection has been established, ${ }^{1}$ the long-term health impacts of fecal microbiota transplantation are still under investigation. Emerging research suggests the intestinal microbiota may influence various metabolic, mental health, autoimmune and other disease states. ${ }^{5}$ Patients should be counselled on these potential risks.

\section{References}

1. Moayyedi $\mathrm{P}$, Yuan $\mathrm{Y}$, Baharith $\mathrm{H}$, et al. Faecal microbiota transplantation for Clostridium difficile-associated diarrhoea: a systematic review of randomised controlled trials. Med J Aust 2017;207:166-72

2. Hota SS, Sales V, Tomlinson G, et al. Oral vancomycin followed by fecal transplantation versus tapering oral vancomycin treatment for recurrent Clostridium difficile infection: an openlabel, randomized controlled trial. Clin Infect Dis 2017;64: 265-71.

3. Guidance document: fecal microbiota therapy used in the treatment of Clostridium difficile infection not responsive to conventional therapies. Ottawa: Health Canada; 2015 (modified 2016 Aug. 12). Available: www.canada.ca/en/health-canada/services /drugs-health-products/biologics-radiopharmaceuticals-genetic -therapies/applications-submissions/guidance-documents/ regulation-fecal-microbiota-therapy-treatment-difficile-infections .html (accessed 2017 Dec. 5)

4. OpenBiome Quality and Safety Program. Somerville (MA) OpenBiome. Available: www.openbiome.org/safety (accessed 2017 Dec. 5).

5. Lynch S, Pederson O. The human intestinal microbiome in health and disease. N Engl J Med 2016;375:2369-79.

Competing interests: Susy Hota has received a grant and honoraria from serving as a consultant and on the advisory board of Cubist Pharmaceuticals, owned by Merck. Susan Poutanen has received honoraria for serving on advisory boards for Merck, Paladin Labs and Accelerate Diagnostics, and has received honoraria for speaking events associated with Merck. She has also received research support from Accelerate Diagnostics, bioMérieux, and Bio-Rad.

This article has been peer reviewed.

Affiliations: Department of Infection Prevention and Control (Hota), University Health Network; Department of Medicine (Hota), University of Toronto; Department of Microbiology (Poutanen), University Health Network and Mount Sinai Hospital; Department of Laboratory Medicine and Pathobiology (Poutanen), University of Toronto, Toronto, Ont.

Correspondence to: Susy Hota, susy.hota@uhn.ca 